11,05

\title{
Дальний порядок в двумерных $O(n)$-моделях, индуцированный случайными полями, и состояние Имри-Ма
}

\author{
(C) А.А. Берзин ${ }^{1}$, А.И. Морозов ${ }^{2, \uparrow}$, А.С. Сигов ${ }^{1}$ \\ ${ }^{1}$ МИРЭА - Российский технологический университет, \\ Москва, Россия \\ ${ }^{2}$ Московский фризико-технический институт (национальный исследовательский университет), \\ Долгопрудный, Московская обл., Россия \\ I E-mail: mor-alexandr@yandex.ru
}

Поступила в Редакцию 6 сентября 2019 г.

В окончательной редакции 6 сентября 2019 г.

Принята к публикации 24 сентября 2019 г.

Рассмотрено влияние дефектов типа „случайное локальное поле“ с анизотропным распределением случайных полей на двумерные модели с непрерывной симметрией векторного параметра порядка. В случае слабой анизотропии случайных полей с понижением температуры имеет место плавный переход от парафазы с динамическими флуктуациями параметра порядка к фазе Имри-Ма со статическими флуктуациями, вызванными флуктуациями случайного поля дефектов. В случае сильной анизотропии случайных полей дефекты приводят к эффективному уменьшению числа компонент параметра порядка и возникновению при конечной температуре фазового перехода в упорядоченное состояние.

Ключевые слова: дефекты типа „случайное локальное поле“; двумерные $O(n)$-модели; фазовая диаграмма; фаза Имри-Ма.

DOI: 10.21883/FTT.2020.02.48880.575

\section{1. Введение}

Хорошо известно, что в двумерных системах с непрерывной симметрией $n$-компонентного векторного параметра порядка $(O(n)$-модели) дальний порядок при конечной температуре отсутствует. В двумерной $X-Y$-модели $(n=2)$ при конечной температуре имеет место фазовый переход из парамагнитной фазы с экспоненциально спадающей корреляционной функцией параметра порядка в фазу Березинского-Костерлица-Таулеса (БКТ) со степенным характером убывания корреляционной функции [1-3]. В двумерной модели Гейзенберга $(n=3)$ парамагнитная фаза существует при всех отличных от нуля температурах [3]. Введение слабой одноионной анизотропии приводит при низкой температуре к изменению критического поведения системы. В случае анизотропии типа „легкая ось“, как показано в работе [4], имеет место фазовый переход в ферромагнитную фазу. В случае анизотропии типа „легкая плоскость“ происходит фазовый переход из парамагнитной фазы в фазу БКТ. Аналогичное рассмотрение влияния слабой анизотропии типа „легкая ось“ на поведение двумерной $X-Y$-модели не проводилось.

Как было показано в наших работах [5-7], введение в систему с $O(n)$ симметрией параметра порядка дефектов типа „случайное локальное поле“ с анизотропным распределением направлений случайных полей дефектов в $n$-мерном пространстве параметра порядка индуцирует во втором порядке по случайному полю эффективную глобальную анизотропию, которая стремится сориентировать параметр порядка перпендикулярно преиму- щественному направлению случайных полей. Именно этой анизотропией объясняется появление при конечной температуре дальнего порядка в двумерной $X-Y$-модели в результате введения в систему дефектов с коллинеарными направлениями локальных полей $[8,9]$.

Кроме того, примеси типа „случайное локальное по-

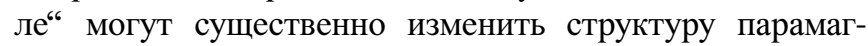
нитной фазы, переведя ее в неоднородное состояние Имри-Ма [10], в котором параметр порядка следует за пространственными флуктуациями направления случайного поля, созданного дефектами.

Данная работа посвящена построению фазовой диаграммы двумерных $X-Y$-модели и модели Гейзенберга со слабой анизотропией, индуцированной анизотропным распределением случайных полей дефектов.

\section{2. Система классических спинов}

Энергия обменного взаимодействия $n$-компонентных локализованных спинов $\mathbf{s}_{i}$ фиксированной единичной длины (длина вектора может быть включена в соответствующие константы взаимодействия или поля), образующих двумерную квадратную решетку, в приближении взаимодействия ближайших соседей имеет вид

$$
W_{e x}=-\frac{1}{2} J \sum_{i, \delta} \mathbf{s}_{i} \mathbf{s}_{i+\delta},
$$

где $J$ - обменный интеграл, суммирование по $i$ ведется по всей решетке спинов, а по $\delta$ - по ближайшим к данному спину соседям. 
При наличии в системе одноионной анизотропии соответствующая энергия записывается в виде

$$
W_{a n}=\frac{1}{2} K \sum_{i}\left(s_{i}^{\alpha}\right)^{2},
$$

где $K$ - константа анизотропии, $s_{i}^{\alpha}-$ одна из $n$ компонент $i$-го спина, суммирование по $i$ ведется по всей решетке спинов.

Энергия взаимодействия спинов со случайными локальными полями дефектов равна

$$
W_{d e f}=-\sum_{l} \mathbf{s}_{l} \mathbf{h}_{l}
$$

суммирование ведется по случайно расположенным в узлах решетки дефектам, $\mathbf{h}_{l}$ - локальное поле $l$-дефекта, a плотность распределения случайных локальных полей $\mathbf{h}$ в спиновом пространстве (пространстве параметра порядка) обладает свойством $\rho(\mathbf{h})=\rho(-\mathbf{h})$, что обеспечивает отсутствие в бесконечной системе среднего поля.

\section{3. Бездефектная двумерная модель Гейзенберга со слабой анизотропией}

Эта задача была рассмотрена в работе [4]. В случае анизотропии типа „легкая ось“ $(K<0)$ система переходит в класс моделей Изинга, и в ней возникает дальний порядок при температуре $T_{c}$, равной [4]

$$
T_{c} \approx \frac{4 \pi J}{\ln \left(\frac{J}{|K|}\right)} .
$$

При $T \sim T_{c}$ происходит эффективное уменьшение $n$ с трех до единицы и в системе возникает дальний порядок.

Выражение (4) легко получить из простых энергетических соображений. Для этого приравняем по абсолютному значению $T_{c}$ и энергию анизотропии $E_{c} \sim K \xi^{2}$ скоррелированной при $T<J$ области с радиусом, равным радиусу корреляции $\xi$. Радиус корреляции двумерной модели Гейзенберга $\xi$ дается формулой [3]

$$
\xi=\exp \left(\frac{2 \pi J}{T}\right) .
$$

Решая получившееся уравнение для переменной $T_{c}$

$$
T_{c}=|K| \exp \left(\frac{4 \pi J}{T_{c}}\right) .
$$

методом итераций и пренебрегая численным множителем порядка единицы под логарифмом, получаем в первом приближении формулу (4).

Данный метод оценки $T_{c}$ хорошо работает и при исследовании систем с сильно анизотропным обменным взаимодействием, например, для нахождения критической температуры квазиодномерной модели Изинга [11].
В этом случае нужно приравнивать к $T_{c}$ не энергию анизотропии, а энергию слабого обменного взаимодействия между скоррелированными одномерными областями соседних нитей, вдоль которых имеет место сильное обменное взаимодействие.

Если же в двумерной модели Гейзенберга возникает слабая анизотропия типа „легкая плоскость“, то при температуре $T_{c}$, задаваемой формулой (4), происходит фазовый переход в фазу БКТ [4], а эффективное число компонент параметра порядка уменьшается с трех до двух.

\section{4. Бездефектная двумерная $X-Y$-модель со слабой анизотропией}

В данной модели с $n=2$ возможна только анизотропия типа „легкая ось“. Воспользуемся и для данной модели описанным в предшествующем разделе методом оценки $T_{c}$. Согласно [3]

$$
\xi=\exp \left(b \tau^{-1 / 2}\right)
$$

где $\tau=\left(T-T_{B K T}\right) / T_{B K T}$, а $T_{B K T}=\pi J / 2-$ температура перехода из парамагнитной фазы в фазу БКТ в отсутствие анизотропии. Из условия $\left|E_{c}\right| \sim T_{c}$ находим в первом приближении, считая, что $T_{c} \sim T_{B K T}$,

$$
\tau_{c} \approx \frac{4 b^{2}}{\ln ^{2} \frac{J}{|K|}} \ll 1 .
$$

Таким образом, наличие слабой анизотропии приводит к тому, что при температуре $T_{c}=\left(1+\tau_{c}\right) T_{B K T}$ в системе происходит фазовый переход в фазу с дальним порядком, и фаза БКТ не возникает. Поскольку в реальных кристаллических системах всегда присутствует слабая анизотропия, индуцированная симметрией кристаллической решетки, то экспериментальное наблюдение фазы БКТ в таких системах вряд ли возможно.

\section{5. Температура $\tilde{T}$ возникновения фазы Имри-Ма}

В отличие от рассмотренных в разделах 3 и 4 систем, в которых одноионная анизотропия присутствовала в бездефектной системе, в данном разделе будет рассмотрен случай, когда анизотропия индуцируется дефектами типа „случайное локальное поле“, то есть в энергии классических спинов присутствуют только слагаемые (1) и (3). Но данные дефекты порождают не только анизотропию, но и флуктуации случайного поля, способствующие разрушению дальнего порядка.

Переход от поведения, присущего парамагнитной фазе чистой системы, к поведению, характерному для неупорядоченной фазы Имри-Ма, происходит при температуре $\tilde{T}$, которая находится из условия равенства 
радиуса корреляции чистой системы $\xi$ и оптимальной длины $L^{*}$ статических флуктуаций параметра порядка в фазе Имри-Ма. При $T>\tilde{T}$ выполнено условие $\xi<L^{*}$ и в системе наблюдаются динамические тепловые флуктуации параметра порядка, характерные для чистой системы. При $T<\tilde{T}$ справедливо соотношение $\xi>L^{*}$ и статические флуктуации случайного поля „замораживают“ эти динамические флуктуации. Имеет место явление, родственное явлению стеклования.

Согласно работе [12], в случае двумерной системы по порядку величины

$$
L^{*} \approx\left(\frac{J^{2}}{x\left\langle\mathbf{h}_{l}^{2}\right\rangle}\right)^{1 / 2}
$$

Здесь $x$ - безразмерная концентрация примесей (их число на одну ячейку), а угловые скобки обозначают усреднение по полям всех дефектов.

В случае двумерной модели Гейзенберга из условия $\xi=L^{*}$ получаем оценку для величины $\tilde{T}$

$$
\tilde{T} \approx \frac{2 \pi J}{\ln L^{*}} \approx \frac{4 \pi J}{\ln \left(\frac{J^{2}}{x\left\langle\mathbf{h}_{l}^{2}\right\rangle}\right)}=\frac{4 \pi J}{\ln \left(\frac{J}{K_{c r}}\right)},
$$

где $K_{c r} \sim x\left\langle\mathbf{h}_{l}^{2}\right\rangle / J-$ критическое значение глобальной анизотропии типа „легкая ось“, делающее существование фазы Имри-Ма энергетически невыгодным [12].

В случае двумерной $X-Y$-модели из этого же условия находим соответствующее замораживанию флуктуаций значение $\tau$

$$
\tilde{\tau} \approx \frac{4 b^{2}}{\ln \left(\frac{J^{2}}{x\left\langle\mathbf{h}_{l}^{2}\right\rangle}\right)} \approx \frac{4 b^{2}}{\ln ^{2}\left(\frac{J}{K_{c r}}\right)} \ll 1 .
$$

Сама температура перехода в состояние Имри-Ма $\tilde{T}=(1+\tilde{\tau}) T_{B K T} \sim T_{B K T}$ оказывается близка к температуре перехода в чистой системе. Аналогичная ситуация имеет место в трехмерных $O(n)$-моделях, где $\tilde{T}$ порядка температуры возникновения дальнего порядка в чистой системе.

\section{6. Фазовая диаграмма двумерной модели Гейзенберга с дефектами типа „случайное локальное поле“}

Сравнивая формулы (4) и (10), легко увидеть, что полученное в работе [12] условие подавления неупорядоченного состояния Имри-Ма $|K|>K_{c r}$ эквивалентно условию $T_{c}>\tilde{T}$. В противном случае с понижением температуры при $T \sim \tilde{T}$ происходит переход из парафазы в фазу Имри-Ма, описанный в предшествующем разделе. Рассмотрим фазовую диаграмму в области $T_{c}>\tilde{T}$.

$\mathrm{C}$ понижением температуры в случае индуцированной дефектами анизотропии типа ,легкая ось“ при $T=T_{c}$ происходит переход к эффективно однокомпонентному параметру порядка, а в случае анизотропии типа „легкая плоскость“ - переход к эффективно двухкомпонентному параметру порядка. Поскольку, как показано в работе [13] (смотри также обзор [14]), низшая критическая размерность для модели Изинга с дефектами типа „случайное локальное поле“ равна двум, то переход к однокомпонентному параметру порядка в двумерной системе сопровождается возникновением ферромагнитной фазы, то есть имеет место фазовый переход парамагнетик-ферромагнетик.

Если при $T=T_{c}$ переход произошел к эффективно двухкомпонентному параметру порядка, то для изучения поведения возникшей системы нужно спроектировать случайные поля дефектов на легкую плоскость, рассчитать параметры $\left|K^{\prime}\right|, K_{c r}^{\prime}, T_{c}^{\prime}$ и $\tilde{T}^{\prime}$ в системе с меньшим числом компонент параметра порядка и исследовать часть фазовой диаграммы $X-Y$-модели с дефектами типа „случайное локальное поле“, отвечающую области температур $T<T_{c}$.

В работе [7] было получено уравнение самосогласования для глобальной эффективной анизотропии, создаваемой анизотропным распределением случайных полей в пространстве параметра порядка двумерной системы

$$
|K|=\frac{x}{4 \pi J}\left(p^{\max }-p^{\min }\right) \ln \frac{4 \pi J}{|K|},
$$

где $p$ - квадратичная форма относительно компонент единичного вектора $\mathbf{s}_{0}$, параллельного среднему значению спина,

$$
p=\sum_{\alpha=1}^{n} s_{0 \alpha}^{2}\left\langle h_{l \alpha}^{2}\right\rangle
$$

a $p^{\max }, p^{\min }-$ максимальное и минимальное значение этой формы относительно всех возможных ориентаций $\mathbf{s}_{0}$ в $n$-мерном пространстве параметра порядка.

В случае компланарного и изотропного в выделенной плоскости распределения случайных полей в системе возникает легкая ось, перпендикулярная этой плоскости. Уравнение самосогласования для константы анизотропии (12) принимает вид [7]

$$
|K|=\frac{x\left\langle\mathbf{h}_{l}^{2}\right\rangle}{8 \pi J} \ln \frac{4 \pi J}{|K|} .
$$

Решая его методом итераций, получаем в первом приближении

$$
|K|=\frac{x\left\langle\mathbf{h}_{l}^{2}\right\rangle}{8 \pi J} \ln \frac{32 \pi^{2} J^{2}}{x\left\langle\mathbf{h}_{l}^{2}\right\rangle} .
$$

В случае $T_{c}>\tilde{T}$ параметр порядка в ферромагнитной фазе ориентирован коллинеарно легкой оси, его крупномасштабные статические флуктуации подавлены, имеют место только локальные отклонения спинов вблизи дефектов. При $T_{c}<\tilde{T}$ в фазе Имри-Ма спины лежат в той же плоскости, что и случайные поля. 


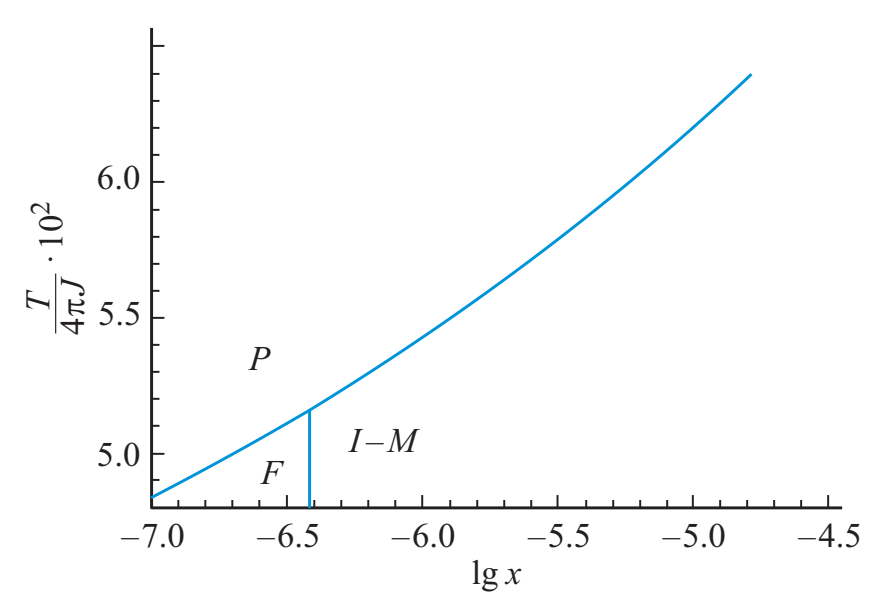

Рис. 1. Фазовая диаграмма двумерной модели Гейзенберга c анизотропией типа „легкая ось“, индуцированной дефектами типа „случайное локальное поле“, при $J^{2} /\left\langle\mathbf{h}_{l}^{2}\right\rangle=100$ : $P$ - парамагнитная фаза, $F-$ ферромагнитная фаза, $I-M-$ неупорядоченная фаза Имри-Ма.

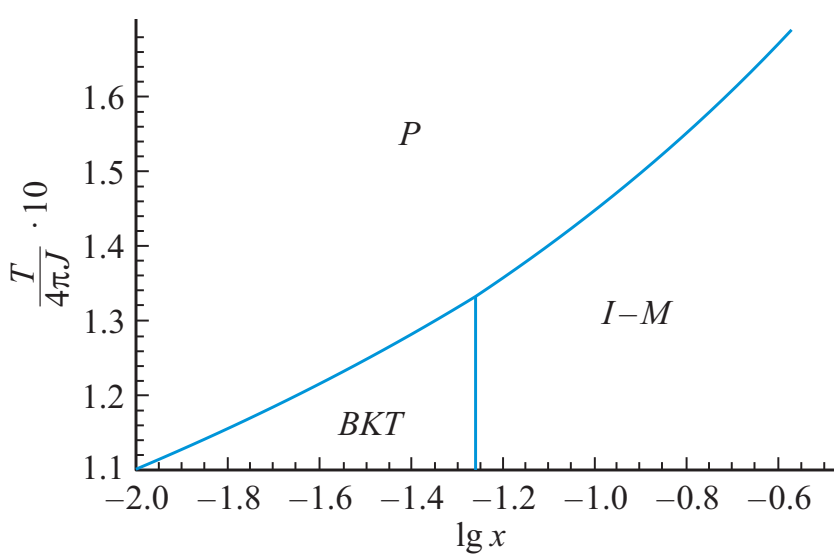

Pис. 2. Фазовая диаграмма двумерной модели Гейзенберга с анизотропией типа „легкая плоскость“, индуцированной дефектами типа „случайное локальное поле“, при $J^{2} /\left\langle\mathbf{h}_{l}^{2}\right\rangle=100$ : $P-$ парамагнитная фаза, $B K T-$ фаза БКТ, $I-M-$ неупорядоченная фаза Имри-Ма.

Условию $T_{c}>\tilde{T}$ при $J^{2} /\left\langle\mathbf{h}_{l}^{2}\right\rangle=100$ соответствует область концентраций $x<x_{c}=4 \cdot 10^{-7}$. Таким образом, даже при данном распределении направлений случайных полей, оптимальном для создания глобальной анизотропии, для всех реальных концентраций дефектов будет наблюдаться фаза Имри-Ма. Фазовая диаграмма двумерной модели Гейзенберга с дефектами типа „случайное локальное поле“ и анизотропией типа ,легкая ось“ приведена на рис. 1.

В случае, когда все случайные поля коллинеарны оси $z$ декартовой ортогональной системы координат, в спиновом пространстве возникает легкая плоскость $x y$. При этом проекции случайных полей на легкую плоскость равны нулю, что делает возникшую систему с двухкомпонентным параметром порядка эквивалентной чистой $X-Y$-модели. Поэтому при температуре $T_{c}$, задаваемой формулой (4) со значением $|K|$, равным [7]

$$
|K|=\frac{x\left\langle\mathbf{h}_{l}^{2}\right\rangle}{4 \pi J} \ln \frac{16 \pi^{2} J^{2}}{x\left\langle\mathbf{h}_{l}^{2}\right\rangle},
$$

происходит фазовый переход в фазу БКТ, в которой спины лежат в легкой плоскости.

Условию $T_{c}>\tilde{T}$ при $J^{2} /\left\langle\mathbf{h}_{l}^{2}\right\rangle=100$ соответствует область концентраций $x<x_{c}=5.5 \cdot 10^{-2}$. Таким образом, переход из фазы БКТ в фазу Имри-Ма по мере роста концентрации дефектов происходит при заметной критической концентрации. Фазовая диаграмма двумерной модели Гейзенберга с дефектами типа „случайное локальное поле“ и анизотропией типа ,легкая плоскость“" приведена на рис. 2.

\section{7. Фазовая диаграмма двумерной $X-Y$-модели с дефектами типа „случайное локальное поле“}

Аналогично рассмотрению, проведенному в предшествующем разделе, условие подавления неупорядоченного состояния Имри-Ма $|K|>K_{c r}$ эквивалентно соотношению $\tau_{c}>\tilde{\tau}$. Если же $|K|<K_{c r}$, то при $\tau \sim \tilde{\tau}$ парамагнитная фаза переходит в состояние Имри-Ма.

В случае анизотропного распределения направлений случайных полей, при котором все $\mathbf{h}_{l}$ коллинеарны, в системе появляется легкая ось, перпендикулярная направлению случайных полей, а значение константы анизотропии дается формулой (16). Поскольку проекции случайных полей на легкую ось равны нулю, то возникающая система с однокомпонентным параметром порядка эквивалентна чистой двумерной модели Изинга. Поэтому при $\tau=\tau_{c}$ в системе происходит фазовый переход из парамагнитной фазы в ферромагнитную, структура которой аналогична структуре ферромагнитной фазы, описанной в предшествующем разделе. Условие $\tau_{c}>\tilde{\tau}$ выполнено в области концентраций $x<x_{c}=5.5 \cdot 10^{-2}$. Однако и при больших концентрациях, когда $\tau_{c}<\tilde{\tau}$ и разупорядочивающее действие случайных полей преобладает, в системе существует дальний порядок. Причины этого рассмотрены нами в работе [15]. Поскольку все случайные поля коллинеарны некоторой прямой в двумерном пространстве параметра порядка (выберем ее в качестве оси $\xi$ декартовой ортогональной системы координат $(\xi, \eta))$, то флуктуация случайного поля в области с характерным линейным размером $L^{*}$ направлена либо параллельно, либо антипараллельно оси $\xi$. При переходе от области с одним направлением поля к соседней области с противоположным направлением поля параметр порядка совершает разворот на $180^{\circ}$. Энергия неоднородного обмена будет заведомо меньше, если этот разворот во всей решетке спинов будет происходить по одной и той же дуге, проходящей, например, через положительную полуось $\eta$. При этом в системе возникнет 


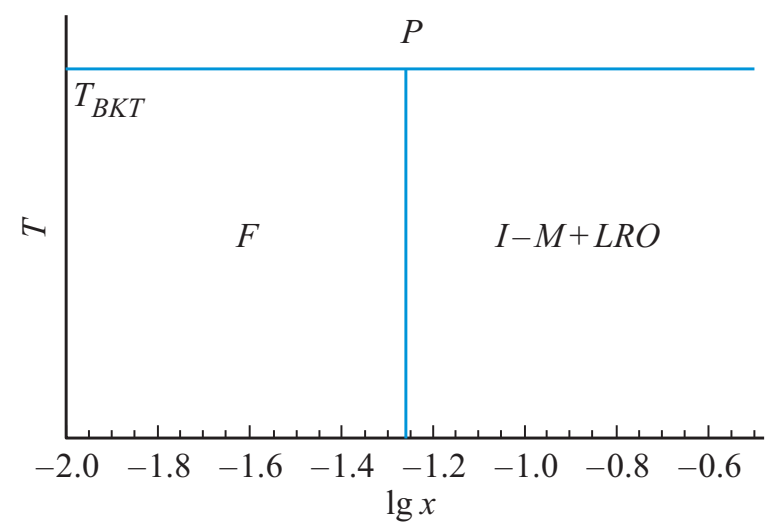

Рис. 3. Фазовая диаграмма двумерной $X-Y$-модели с коллинеарными направлениями случайных полей дефектов при $J^{2} /\left\langle\mathbf{h}_{l}^{2}\right\rangle=100: P-$ парамагнитная фаза, $F-$ ферромагнитная фаза, $I-M+L R O-$ фаза Имри-Ма с дальним порядком.

среднее значение векторного параметра порядка, параллельное оси $\eta$. Это объясняет результаты моделирования работ $[8,9]$, авторы которых ввели случайное поле в каждую ячейку $(x=1)$ и продемонстрировали наличие дальнего порядка, индуцированного случайными полями (random field induced order). Среднее значение параметра порядка даже в основном состоянии далеко от насыщения, так как флуктуации случайного поля приводят к существенным статическим флуктуациям параметра порядка. Имеет место сосуществование фазы Имри-Ма и ферромагнитной фазы. Следует отметить, что данная комплексная фаза представляется нам уникальной. Во всей области концентраций дефектов фазовый переход в ферромагнитную фазу или фазу Имри-Ма с дальним порядком происходит при $T \sim T_{B K T}$. Фазовая диаграмма двумерной $X-Y$-модели с дефектами типа „случайное локальное поле“ приведена на рис. 3.

\section{8. Выводы}

В заключение сформулируем основные выводы работы:

1. Анизотропное распределение случайных полей дефектов, создавая глобальную анизотропию, эффективно уменьшает число компонент параметра порядка. В случае двумерных $O(n)$-моделей это может привести к возникновению в системе дальнего порядка при конечной температуре.

2. В случае бездефектной двумерной $X-Y$-модели появление сколь угодно слабой анизотропии в двумерном пространстве параметра порядка полностью исключает появление фазы Березинского-Костерлица-Таулеса и приводит к появлению фазы с дальним порядком.

3. Поскольку дефекты типа „случайное локальное поле“ создают статические флуктуации случайного поля, способствующие разупорядочению системы спинов, то в двумерной модели Гейзенберга с указанными дефектами и анизотропией типа „легкая ось“ при их концентрации, превышающей $4 \cdot 10^{-7}$, с понижением температуры происходит плавный переход от динамических флуктуаций параметра порядка, характерных для чистой системы, к фазе Имри-Ма со статическими флуктуациями параметра порядка, следующими за флуктуациями направления случайного поля.

4. В случае двумерной $X-Y$-модели и коллинеарных случайных полей дефектов при температуре $T \sim T_{B K T}$ и концентрации дефектов, меньшей $5.5 \cdot 10^{-2}$, происходит фазовый переход из парамагнитной фазы в ферромагнитную, а при большей концентрации дефектов - в фазу Имри-Ма с дальним порядком.

\section{Финансирование работы}

Работа поддержана Министерством науки и высшего образования РФ (госзадание, проект № 8.1183.2017ПЧ).

\section{Конфликт интересов}

Авторы заявляют, что у них нет конфликта интересов.

\section{Список литературы}

[1] В.Л. Березинский. ЖЭТФ 59, 907 (1971); 61, 1144 (1972).

[2] J.M. Kosterlitz, D.G. Thouless. J. Phys. C 6, 1181 (1973).

[3] Ю.А. Изюмов, Ю.Н. Скрябин. Статистическая механика магнитоупорядоченных систем. Наука, М. (1987). 268 с.

[4] С.Б. Хохлачев. ЖЭТФ 70, 265 (1976).

[5] А.А. Берзин, А.И. Морозов, А.С. Сигов. ФТТ 58, 1614 (2016).

[6] А.А. Берзин, А.И. Морозов, А.С. Сигов. ФТТ 58, 1783 (2016).

[7] А.А. Берзин, А.И. Морозов, А.С. Сигов. ФТТ 59, 1992 (2017).

[8] B.J. Minchau, R.A. Pelcovits. Phys. Rev. B 32, 3081 (1985).

[9] J. Wehr, A. Niederberger, L. Sanchez-Palencia, M. Lewenstein. Phys. Rev. B 74, 224448 (2006).

[10] Y. Imry, S.-K. Ma. Phys. Rev. Lett. 35, 1399 (1975).

[11] D.J. Scalapino, Y. Ymry, P. Pincus. Phys. Rev. B 11, 2042 (1978).

[12] А.И. Морозов, А.С. Сигов. Письма в ЖЭТФ 90, 818 (2009).

[13] J. Imbrie. Phys. Rev. Lett. 53, 1747 (1984).

[14] Вик. С. Доценко. УФН 165, 481 (1995)

[15] А.А. Берзин, А.И. Морозов. ФТТ 57, 2155 (2015).

Редактор К.В. Емцев 